\title{
Interaction Of A Stellar Magnetic Field With A Turbulent Accretion Disk
}

\author{
Anne Bardou and Jean Heyvaerts \\ Observatoire de Strasbourg, 11 rue de l'Université, 67000 Strasbourg, \\ France
}

\begin{abstract}
Magnetized objects surrounded by a turbulent and keplerian accretion disk are considered. In such systems, magnetic field lines are embedded into the disk. The magnetic field of the central object is assumed to be dipolar in the absence of the accretion disk. In the presence of a turbulent accretion disk, it is shown that the interaction with the disk stretches magnetic field lines along the disk and that most of the non-magnetospheric magnetic flux is expelled outside the disk.
\end{abstract}

\section{Introduction}

$\mathrm{X}$-ray binaries and classical $\mathrm{T}$-tauri stars are generally assumed to have a central magnetized object surrounded by an accretion disk. Such systems thus show an interaction between the stellar magnetic field and the disk. The purpose of this work is to study this interaction. The stellar magnetic field is taken to be initially dipolar and the accretion disk is considered to be thin, keplerian and turbulent.

The rotation of the disk creates an azimuthal component in an initially dipolar magnetic field. The twist of the magnetic field is defined as the ratio between the azimuthal component $B_{\phi}$ of the magnetic field and its vertical component $B_{z}$ in the disk. It is given by

$$
\frac{B_{\phi}}{B_{z}}=\frac{1}{4 \alpha} \frac{R}{h}
$$

where an $\alpha$-viscosity prescription is considered ( $\alpha \leq 1), h$ is the half-thickness of the disk and $R$ is the radial coordinate. For a thin accretion disk $(h / R \ll 1)$ with $\alpha \leq 1$ the twist becomes very large. The configuration is therefore unstable and a dipolar magnetic field does not correspond to a stationary solution. In this paper we present a stationary model for the stellar magnetic field in the presence of a turbulent accretion disk. Part of this work is discussed in more detail in Bardou \& Heyvaerts (1996).

\section{The solution}

Theorems about magnetic field lines sheared by a velocity field exist (Aly 1984, 1991) but cannot be applied here. The reason is that the magnetic Reynolds 
number $R_{M}$ based on the radial velocity is very small. Consequently the field lines are not fixed in the radial direction. In fact, one has

$$
R_{M}=\frac{\nu_{\star}}{\eta_{\star}} \times \frac{h}{R}
$$

where $\nu_{\star}$ is the turbulent viscosity and $\eta_{\star}$ is the turbulent magnetic diffusivity. In the absence of a disk wind these two quantities are linked by the Prandlt number $P$. We thus obtain

$$
R_{M}=\frac{1}{P} \times \frac{h}{R} \ll 1
$$

In other words, the magnetic diffusivity is very large in the radial direction (in contrast to the azimuthal direction) and hence the magnetic field lines remain perpendicular to the disk. Due to large diffusivity the theorems of Aly (1984, 1991) are not applicable.

\subsection{The intuitive solution}

In this section, we use a Gedankenexperiment to intuitively predict the behaviour of the magnetic field lines in a turbulent accretion disk (Figure 1).

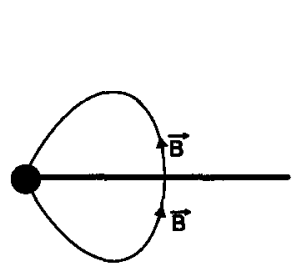

(1a)
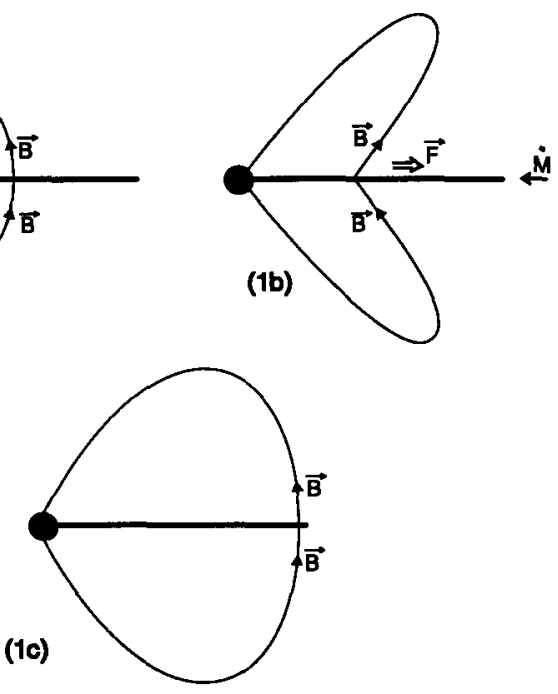

Figure 1. Qualitative evolution of the magnetic field in a turbulent accretion disk. See text for details.

We first consider an initial dipolar magnetic field configuration without accretion of matter (Figure 1a). We then switch on the accretion of matter and the disk is regarded as non-diffusive. Thus the field lines are dragged inwards by the accretion and sheared azimuthally by the rotation of the disk. The latter causes the dipolar field structure to inflate according to the theorems of Aly. The field line curvature generates a force that tries to push the footpoints of the poloidal magnetic field outwards (Figure 1b). When, in a third step, we take 
into account the diffusivity in the disk the footpoints move radially outwards and we end up with an inflated magnetic structure (Figure 1c). In the following, a demonstration of this behaviour will be given.

\subsection{Numerical calculations}

Considering a force-free magnetic configuration in the corona (the zone between the central object and the disk) we have solved the Grad-Shafranov equation using an iterative method. We introduced a parameter which allows us to control the twist of the system. A typical result is shown in Figure 2.

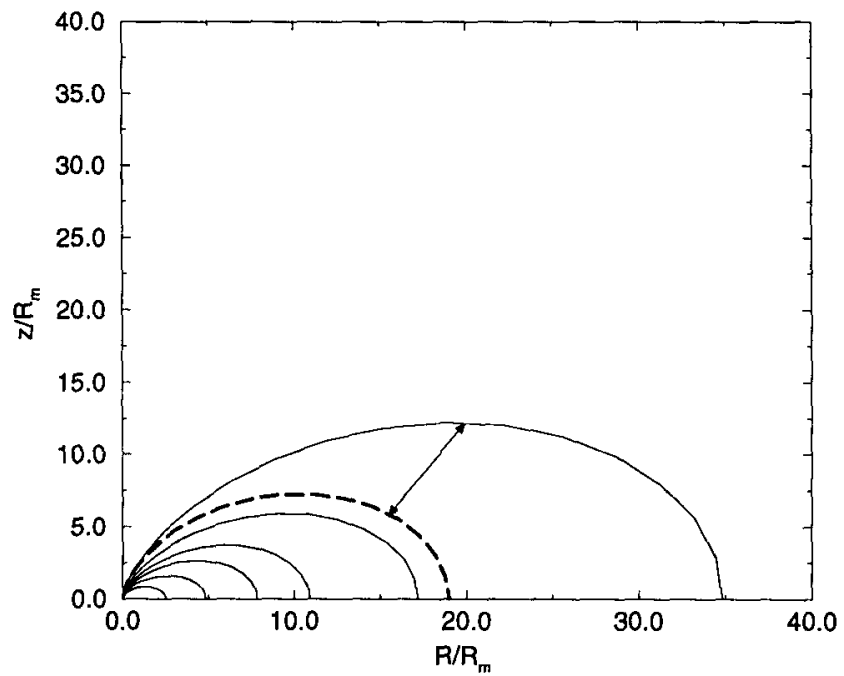

Figure 2. Magnetic field lines for a twist of $8 \cdot 10^{-5}$ times its real value for $\alpha=0.04$. The dashed line curve gives one dipolar shape for comparison.

It can be seen that the inflation is already large (a factor 2 on the value of the intersection radius between the magnetic field line and the disk) even if the twist is still small. This calculation is numerically limited to small twist. We therefore looked for an analytical solution using a self-similar assumption.

\subsection{The self-similar solution}

We start with the Graf-Shafranov equation written in spherical coordinates

$$
\frac{1}{r}\left[\frac{\partial}{\partial r}\left(\frac{1}{\sin \theta} \frac{\partial a}{\partial r}\right)+\frac{\partial}{\partial \theta}\left(\frac{1}{r^{2} \sin \theta} \frac{\partial a}{\partial \theta}\right)\right]=-\frac{I I^{\prime}}{r \sin \theta}
$$

where $r$ is the spherical radius, $\theta$ is the angle with the polar axis, $a$ is the usual flux function constant along one particular field line, $I=R B_{\phi}$ and $I^{\prime}=\mathrm{d} I / \mathrm{d} a$.

The self-similar assumption is equivalent to writing the flux function as

$$
a=r^{-p} f(\mu)
$$


with $\mu=\cos \theta$ and $p$ a parameter to be determined (Lynden-Bell \& Boily 1994). As boundary conditions we take $f(\mu=1)=0$ and $f(\mu=0)=1$. In addition, we require that the lines stay perpendicular to the disk i.e. $f^{\prime}(\mu=0)=1$. With the Ansatz (5) the Grad-Shafranov equation (4) can then be solved. The parameter $p$ which describes the distribution of the flux on the surface of the disk becomes

$$
p=\sqrt{\frac{1}{1+\left(B_{\phi} / B_{z}\right)^{2}}} \sim\left|\frac{B_{\phi}}{B_{z}}\right|_{d i s k}^{-1} .
$$

It can easily be seen that if $p=1$ we get a dipolar magnetic field, if $p \ll 1$ the line are inflated and stretched along the radial axis and if $p \rightarrow 0$ then the lines open. What is the value of $p$ in a turbulent accretion disk? We have shown that (Bardou \&Heyvaerts 1996)

$$
\frac{B_{\phi}}{B_{z}}=\frac{2.10^{2}}{\alpha}\left(\frac{M_{\star}}{M_{\odot}}\right)^{3 / 8}\left(\frac{10^{-8} M_{\odot} / y r}{\dot{M}}\right)^{1 / 8}\left(\frac{10^{8} m}{R}\right)^{1 / 8}\left(1-\sqrt{\frac{R_{\star}}{R}}\right)^{-1 / 8}\left(1-\frac{\Omega_{\star}}{\Omega_{D}}\right)
$$

It can also be argued that

$$
\frac{B_{\phi}}{B_{z}} \geq\left(\frac{B_{\phi}}{B_{z}}\right)_{\Omega_{\star}=0}
$$

Thus for typical X-ray binaries parameters we get $p=\alpha \times 10^{-2}$ and $p=\alpha \times 10^{-1}$ typical T-tauri parameters. The value of $p$ is therefore small in both cases and magnetic field lines are stretched and elongated in the horizontal direction. A large part of the flux will eventually be pushed beyond the external radius of the disk. We estimate that $91 \%$ to $99.9 \%$ of the flux is expelled in the case of $\mathrm{X}$-ray binaries and $54 \%$ to $99 \%$ in the case of T-tauri (for $0.01 \leq \alpha \leq 0.1$ ). Thus we see that most of the non-magnetospheric magnetic flux is expelled outside a turbulent accretion disk for a large range of $\alpha$.

\section{Conclusion}

We have stressed that, in a turbulent accretion disk where accretion is dominated by viscous turbulent torques, the radial magnetic diffusivity is large. Due to the azimuthal rotation of the disk, magnetic field lines inflate and become elongated in the radial direction for all reasonable values of $\alpha$. This situation leads to the expulsion of the major part of the non-magnetospheric magnetic flux. In the future, we will try to extend this work to the magnetospheric zone where the rotation is no more keplerian.

\section{References}

Aly, J.J. 1984, ApJ, 283, 349

Aly, J.J. 1991, ApJ, 375, L61

Bardou, A., Heyvaerts, J. 1996, A\&A, 307, 1009

Lynden-Bell, D., Boily, C. 1994, MNRAS, 267, 146 


\section{Discussion}

K. Horne: You have shown us the shape of the field in the poloidal plane, but does not the field also have a toroidal component, because the disk should drag the footpoint forward or backward in the $\phi$ direction?

A. Bardou: First the field lines which are expelled out of the disk do not have anymore twist. Second for the ones which still have their footpoints in the disk the twist $B_{\phi} / B_{z}$ is always less than 1 . Moreover this twist has to be small otherwise the solution wouldn't be an adequate stationary solution.

$J$. $L i$ : Does the value of $\alpha$ change before the field configuration becomes highly non-dipolar?

A. Bardou: I cannot answer this question properly because I do not calculate the time evolution of the magnetic field line but only the stationary solution. 